\title{
Adaptación de la práctica psiquiátrica en instituciones de salud mental públicas y privadas de la Ciudad de Buenos Aires durante la pandemia de COVID-I 9
}

\author{
Adaptation of psychiatric practice in public and private mental health institutions of the \\ City of Buenos Aires during the COVID-19 pandemic
}

\section{Melany Oppel', Sebastián Camino², José María Smith²,Antonella Godoy², Sergio Strejilevich ${ }^{2}$}

\section{Resumen}

Objetivos: Uno de los impactos indirectos más significativos de la pandemia de COVID-19 recaerá sobre la salud mental de la población. En este trabajo relevamos la capacidad de adaptación que han tenido los más representativos Servicios de Salud Mental (SSM) de la Ciudad de Buenos Aires (CABA) frente a este nuevo escenario. Métodos: Se diseñó una encuesta con 10 preguntas cerradas de carácter autoadministrable y anónima. Fue enviada a determinados profesionales de SSM privados y públicos de CABA a 2 meses de iniciada la cuarentena. Resultados: Obtuvimos un total de 38 respuestas, 2 se negaron a responder. 34\% pertenecía al sector privado y $66 \%$ al público. $81 \%$ del total informó haber implementado la atención por telemedicina. $24 \%$ del total informó haber recibido capacitación sobre abordaje de pacientes en este contexto. $69 \%$ perteneciente al sector privado y $12 \%$ al público informó haber recibido capacitación sobre herramientas de telemedicina. $69 \%$ perteneciente al sector privado y $36 \%$ al público informó que su servicio elaboró algún material instructivo para usuarios. $68 \%$ del total refirió que su servicio se ha organizado correctamente. $40 \%$ perteneciente al sector público habría sido reasignado a realizar nuevas tareas. $40 \%$ del total reportó una menor capacidad de atención. Conclusiones: Los SSM de CABA habrían podido migrar su atención a telepsiquiatría aunque con diferencias en el nivel de entrenamiento. Se precisaría mayor capacitación en esta modalidad de atención.

Palabras clave: Psiquiatría - Salud Mental - COVID-I 9 - Telemedicina - Telepsiquiatría.

\section{Abstract}

Background: One of the most significant indirect impacts of the COVID-19 pandemic will be seen on the mental health of the population. On this study, we will take into account the adapting capacity that the most representative mental health services (MHS) of Buenos Aires (BA) City have had as to this new situation. Methods:We designed an online survey including 10 self-administered closed questions, strictly anonymous. It has been sent to targeted professionals who work in public and private MHS of BA after 2 months of the beginning of the lockdown. Results: We got 38 answers. 2 professionals rejected to answer. $34 \%$ belonged to private institutions and $66 \%$ to public ones. $81 \%$ of the total were able to implement online assistance but only $24 \%$ had been trained on how to treat patients in this context. $69 \%$ of the private and $12 \%$ of the public sector professionals informed to have been trained on telemedicine tools. $69 \%$ of the private and $36 \%$ of the public sector professionals informed to have prepared materials for the users on telemedicine resources. $68 \%$ mentioned that their service was properly organized. $40 \%$ of the public sector professionals may have been reassigned to work on tasks related to the pandemic. $40 \%$ of the total informed a reduced capacity of assistance.

RECIBIDO 7/6/202I - ACEPTADO I3/9/2021

1. Médico especialista en Psiquiatría, Magister. Área,Asistencia e investigación en trastornos del ánimo.

2. Médico especialista en Psiquiatría. Área, Asistencia e investigación en trastornos del ánimo. 
Conclusions: The MHS of BA may have been able to migrate their assistance to telemedicine, however we have noticed differences in the training levels. A better capacity of training on this modality might be needed.

Keywords: Psychiatry - Mental Health - COVID-19 - Telemedicine - Telepsychiatry.

\section{Introducción}

Se ha señalado que uno de los impactos indirectos más significativos de la pandemia de COVID-19, recaerá sobre la salud mental (Brooks, 2020). Esto se vería reflejado en tres escenarios posibles. En primer lugar, se ha señalado que aquellas personas que sufrían previamente patologías psiquiátricas podrían experimentar un agravamiento de sus enfermedades (Vindegaard, 2020). Esto se daría, tanto por las dificultades de acceso a los dispositivos de tratamiento determinado por las medidas de aislamiento social, como por el incremento súbito y sostenido del estrés en este contexto. Por otra parte, sería esperable que se observe un incremento en la incidencia de problemas de salud mental en personas que no presentaban antecedentes de este tipo de padecimientos (Moreno, 2020), como consecuencia de las pérdidas humanas y económicas secundarias a la pandemia y el impacto psicológico que determinan las medidas de aislamiento social. Estas situaciones son las que han sido difundidas por los medios como la "Cuarta ola" del impacto en salud por la pandemia. Sumado a esto, se espera que un porcentaje significativo de los profesionales de la salud que hayan trabajado de manera directa con la pandemia sufran consecuencias psicológicas negativas de forma secundaria (Salazar, 2020). Datos recogidos durante las epidemias de SARS y MERS han mostrado que alrededor del $25 \%$ del personal sanitario que trabajó en dicho contexto, sufrió condiciones relacionadas con el burnout (Maunder, 2006).Un estudio de prevalencia realizado en China durante la pandemia actual ha reportado una alta prevalencia de síntomas psiquiátricos en trabajadores de la salud tratando de forma directa pacientes diagnosticados con COVID-19: 50\% reportó síntomas de depresión, 45\% de ansiedad, 34\% de insomnio, y $72 \%$ de distrés (Lai, 2020).

Más allá de que estas predicciones resulten más o menos acertadas, es evidente que la fortaleza y capacidad de adaptación del sistema de salud mental a esta situación emergente debe ser examinada con el fin de afrontar de mejor manera este probable desafío. Dentro de las medidas básicas que han debido adoptar los sistemas de salud mental de todo el mundo, posiblemente la más determinante ha sido la brusca necesi- dad de migrar el sistema de atención ambulatoria a dispositivos de telemedicina (De Girolamo, 2020). Si bien tanto la psiquiatría como la psicología son dos de las disciplinas que mejor se adaptan a esta modalidad y un número significativo de datos han demostrado que la misma es segura y efectiva, su uso estaba reservado a situaciones puntuales, generalmente para la asistencia de poblaciones aisladas (Cowan, 2019) o en situación de catástrofe. Por lo tanto, la brusca emigración a esta modalidad de atención, más allá de los datos que la avalan, ha determinado un desafío para la mayoría de los sistemas de salud mental en el mundo.

La Ciudad Autónoma de Buenos Aires (CABA) en Argentina, es una de las ciudades que ha sido sometida a medidas de aislamiento social más prolongadas durante esta pandemia. Al momento de escribir este reporte se llevan más de 120 días de cuarentena que, si bien ha logrado un enlentecimiento en la curva de contagios, también habría determinado un significativo impacto en la economía y bienestar psicológico de sus habitantes. En esta ciudad, el sistema de salud general y el de salud mental en particular es mixto: público y privado. El sistema privado de salud a su vez es múltiple y heterogéneo ya que está compuesto por aseguradoras privadas de salud y obras sociales sindicales a los que se suman un número no determinado de profesionales que trabajan de manera independiente y fuera de instituciones. La última estimación oficial dio cuenta que el $81 \%$ de la población de CABA tiene acceso al sistema privado de salud, mientras que el 19\% cuenta únicamente con cobertura de salud pública (INDEC, 2010). En este contexto, es de destacar que al momento no existe un sistema integrado de datos que permita monitorear la capacidad y disposición de ambos sistemas, así como tampoco un sistema que integre los datos epidemiológicos del sistema público y privado de salud mental.

El objetivo de este trabajo fue relevar de manera sucinta la forma en que se están adaptando las principales instituciones de salud mental públicas y privadas de esta ciudad frente a la situación de aislamiento social por COVID-19, haciendo foco en la migración a la telemedicina. 


\section{Métodos}

A los fines de realizar el relevamiento se diseñó una encuesta on-line con 10 preguntas cerradas de carácter autoadministrable. Este instrumento fue enviado al menos a dos profesionales de cada una de las principales instituciones privadas y públicas de salud mental elegidas como foco del presente relevamiento, procurando que los mismos fuesen un coordinador o jefe de unidad y el otro un profesional de planta permanente. Dado que los datos a relevar son sensibles y están relacionados con la calidad de las instituciones, se presumió que los informantes podrían ver condicionadas sus respuestas si no se aseguraban las medidas de anonimato. Por esta razón no se consignó la identidad, así como tampoco la institución en la que trabajan, evitándose al mismo tiempo que el sistema on-line (Google forms) recogiera los emails de las personas que contestaran la misma. Esta encuesta se envió durante el mes de mayo, a 2 meses de haber comenzado el aislamiento social preventivo y obligatorio en Argentina.

Para el análisis estadístico se utilizó estadística descriptiva sin realizarse pruebas de comparación cualitativa, dado el tamaño de la muestra.

\section{Resultados}

Obtuvimos un total de 38 respuestas. 2 profesionales se negaron a responder el cuestionario por temor a represalias por parte de su institución, según reportaron. El 34\% de los encuestados informó trabajar en instituciones de salud mental del ámbito privado, mientras que el $66 \%$ en el sector público (de los cuales un 52\% refirió pertenecer a instituciones que brindan asistencia monovalente y un $48 \%$ polivalente). Un $60,5 \%$ re- firió desempeñarse como profesional de planta, 18,5\% como jefe y $16 \%$ como residente. Las respuestas a las preguntas principales se encuentran en la tabla 1.

Adicionalmente se solicitó una valoración respecto de si la capacidad actual de atención de la institución es similar que previo al contexto de aislamiento social, con respuestas similares en los 2 sectores: $40 \%$ del público y $38,5 \%$ del privado reportaron una menor capacidad de atención.

\section{Discusión}

Según nuestro conocimiento, este es el primer relevamiento destinado a tener una visión inicial sobre el modo en que se ha adaptado el sistema de salud mental de la CABA a la situación planteada por la pandemia de COVID-19.

Más de la mitad de los profesionales relevados de ambos sectores opinaron que sus instituciones se habían organizado correctamente respecto de evitar la propagación del virus. Coherente con esto, aunque con asimetrías, las instituciones relevadas habrían podido cambiar su modalidad de atención ambulatoria a telemedicina. Mientras que el $100 \%$ de los profesionales de instituciones privadas informó haber migrado la atención ambulatoria a telemedicina, solo el $70 \%$ de los profesionales del sector público lo informó del mismo modo. Sin embargo, esta asimetría se hace mayor respecto del entrenamiento que habrían recibido los profesionales respecto de esta modalidad de trabajo. Mientras el 69\% de los profesionales del sector privado relevados reportó haber sido instruido sobre el uso de herramientas de telemedicina, solo lo hizo el $12 \%$ de los profesionales del sector público. Asimetría

Tabla I. Resultados principales

\begin{tabular}{|c|c|c|c|c|c|c|}
\hline & \multicolumn{2}{|c|}{$\begin{array}{c}\text { Total } \\
(\mathrm{n}: 38=100 \%)\end{array}$} & \multicolumn{2}{|c|}{$\begin{array}{c}\text { Público } \\
\text { (n: } 25=66 \%)\end{array}$} & \multicolumn{2}{|c|}{$\begin{array}{c}\text { Privado } \\
\text { (n: } 13=34 \%)\end{array}$} \\
\hline & Sí & No & Sí & No & Sí & No \\
\hline $\begin{array}{l}\text { ¿Se ha implementado la telemedicina o video llamada como forma de } \\
\text { atención remota? }\end{array}$ & 81.5 & 18.5 & 72 & 28 & 100 & 0 \\
\hline $\begin{array}{l}\text { ¿Ha sido instruido sobre el uso de herramientas de telemedicina o se } \\
\text { ha elaborado algún tutorial sobre atención remota? }\end{array}$ & 31.5 & 68.5 & 12 & 88 & 69 & 31 \\
\hline $\begin{array}{l}\text { ¿Ha recibido alguna capacitación específica sobre abordaje o asistencia } \\
\text { de pacientes en este contexto? }\end{array}$ & 23.7 & 76.3 & 24 & 76 & 23 & 77 \\
\hline $\begin{array}{l}\text { ¿Su servicio ha elaborado algún material informativo/instructivo para } \\
\text { los usuarios (pacientes)? }\end{array}$ & 47.5 & 52.5 & 36 & 64 & 69 & 31 \\
\hline $\begin{array}{l}\text { En su opinión, ¿en su servicio se ha organizado correctamente la asis- } \\
\text { tencia de los profesionales para reducir la propagación del virus? }\end{array}$ & 68.5 & 31.5 & 60 & 40 & 84.5 & 15.5 \\
\hline $\begin{array}{l}\text { ¿Ha sido reasignado a realizar tareas que normalmente no realiza (por } \\
\text { ej. trabajar en una unidad de pacientes febriles)? }\end{array}$ & 26.3 & 73.7 & 40 & 60 & 0 & 100 \\
\hline
\end{tabular}


similar a la que se encontró en el reporte de la disponibilidad de información para usuarios. En este contexto, el $40 \%$ de los profesionales relevados de salud mental del sector público informo haber sido reasignado a tareas que habitualmente no realizaba, particularmente aquellas que podrían estar relacionadas con la contención de la pandemia y que, a su vez suponen un mayor riesgo de contraer la enfermedad. En cambio, ninguno de los profesionales del sector privado informó haber sido reasignado a tareas no habituales.

El aspecto en el cual se denotó una mayor coherencia entre ambos sectores fue en la disminución de la capacidad de atención de los profesionales: un 40\% de los profesionales de ambos sectores reportó disminución en la capacidad de atención de su servicio, lo cual nos hace suponer que un número de pacientes se ha quedado sin acceso a sus tratamientos.

La telemedicina, y la telepsiquiatría en particular, son modalidades de trabajo que han demostrado ser efectivas, bien recibidas por los usuarios y más costo-efectivas que la atención presencial. Es posible que las circunstancias de la pandemia de COVID-19 que han obligado al uso de esta modalidad, facilite en un futuro que la misma sea usada como recurso habitual y no solo extraordinario. Como ejemplo, en un relevamiento realizado entre los pacientes de nuestro centro, el $88,5 \%$ de los pacientes calificó como buena o muy buena la experiencia con la atención a través de tele-entrevistas y $24 \%$ expresó que prefiere seguir con esta modalidad de atención, el $49 \%$ lo considera muy probable y sólo el $24 \%$ espera volver pronto a las entrevistas presenciales (Strejilevich et al., datos sin publicar).

\section{Conclusiones}

En este trabajo hemos encontrado que, si bien las instituciones de los sectores público y privado habrían podido migrar su modalidad de atención a la telemedicina, el entrenamiento de los profesionales y la información disponible para los usuarios sería significativamente menor en el sector público. Consideramos que este es un dato relevante y sobre el cual debería realizarse un seguimiento ya que el mejor coeficiente de costo efectividad (básicamente por evitar los desplazamientos de los usuarios) que permite la telepsiquiatría, sería especialmente conveniente para los sistemas públicos de atención, donde un número significativo de usuarios no viviría en la cercanía de las instituciones en donde consulta.
El presente reporte tiene como limitación principal que sus datos provienen del informe de profesionales que no fueron seleccionados de forma aleatoria y el hecho de que no se haya podido ligar su información con las instituciones en las que trabajan. Esto presupone un importante sesgo y falta de precisión en la información, pero a la hora de diseñar este relevamiento supusimos que de no hacerlo de este modo, no íbamos a encontrar cooperación por parte de los mismos (hecho que en parte fue corroborado ya que aun, con estas medidas para garantizar la confidencialidad de la información, algunos profesionales prefirieron no brindar la misma). A su vez, las 38 respuestas obtenidas no son una muestra representativa de la totalidad de los servicios de salud mental de la Ciudad de Buenos Aires, tanto del ámbito público como privado.

Finalmente, debe entenderse que un informe de estas características y limitaciones se hace necesario por la falta de un sistema de información adecuado desde donde obtener estos datos.

Conflictos de intereses: el artículo no ha recibido ayuda de ninguna agencia de financiación. Los autores Oppel, Camino, Smith y Godoy no presentan conflicto de interés en el tema expuesto. El autor Strejilevich podría presentar potenciales conflictos de interés por ser speaker de los laboratorios: Jannsen, TEVA y Ariston.

\section{Referencias bibliográficas}

Arango, C. (2020). How mental health care should change as a consequence of the COVID-19 pandemic. The Lancet. Psychiatry, 7(9), 813-824. https://doi.org/10.1016/S2215-0366(20)30307-2

Brooks, S. K., Webster, R. K., Smith, L. E., Woodland, L., Wessely, S., Greenberg, N., \& Rubin, G. J. (2020). The psychological impact of quarantine and how to reduce it: rapid review of the evidence. Lancet (London, England), 395(10227), 912-920. https://doi.org/10.1016/S0140-6736(20)30460-8

Coronavirus Disease 2019. JAMA network open, 3(3), e203976. https://doi. org/10.1001/jamanetworkopen.2020.3976

Cowan, K. E., McKean, A. J., Gentry, M. T., \& Hilty, D. M. (2019). Barriers to Use of Telepsychiatry: Clinicians as Gatekeepers. Mayo Clinic proceedings, 94(12), 2510-2523. https://doi.org/10.1016/j.mayocp.2019.04.018

De Girolamo, G., Cervera, G., Clerici, M., Monzani, E., Spinogatti, F., Starace, F., et al. (2020). Mental Health in the Coronavirus Disease 2019 Emergency-The Italian Response. JAMA Psychiatry, Published Online Apr 30. doi: 10.1001/jamapsychiatry.2020.1276.

Instituto Nacional de Estadística y Censos (INDEC)[Internet]. Censo Nacional de Población, Hogares y Viviendas 2010.https://www.indec.gob.ar/. (Último acceso 20 de Julio de 2021).

Lai, J., Ma, S., Wang, Y., Cai, Z., Hu, J., Wei, N., Wu, J., Du, H., Chen, T., Li, R., Tan, H., Kang, L., Yao, L., Huang, M., Wang, H., Wang, G., Liu, Z., \& Hu, S. (2020). Factors Associated With Mental Health Outcomes Among Health Care Workers Exposed to Coronavirus Disease 2019. JAMA network open, 3(3), e203976. https://doi.org/10.1001/jamanetworkopen.2020.3976 
Moreno, C., Wykes, T., Galderisi, S., Nordentoft, M., Crossley, N., Jones, N., Cannon, M., Correll, C. U., Byrne, L., Carr, S., Chen, E., Gorwood, P., Johnson, S., Kärkkäinen, H., Krystal, J. H., Lee, J., Lieberman, J., López-Jaramillo, C., Männikkö, M., Phillips, M. R., ... Maunder, R. G., Lancee, W. J., Balderson, K. E., Bennett, J. P., Borgundvaag, B., Evans, S., Fernandes, C. M., Goldbloom, D. S., Gupta, M., Hunter, J. J., McGillis Hall, L., Nagle, L. M., Pain, C., Peczeniuk, S. S., Raymond, G., Read, N., Rourke, S. B., Steinberg, R. J., Stewart, T. E., VanDeVelde-Coke, S., ... Wasylenki, D. A. (2006). Long-term psychological and occupational effects of providing hospital healthcare during SARS outbreak. Emerging infectious diseases, 12(12), 1924-1932. https://doi.org/10.3201/eid1212.060584
Salazar de Pablo, G., Vaquerizo-Serrano, J., Catalan, A., Arango, C., Moreno, C., Ferre, F., Shin, J. I., Sullivan, S., Brondino, N., Solmi, M., \& Fusar-Poli, P. (2020). Impact of coronavirus syndromes on physical and mental health of health care workers: Systematic review and meta-analysis. Journal of affective disorders, 275, 48-57. https://doi.org/10.1016/j. jad.2020.06.022

Vindegaard, N., \& Benros, M. E. (2020). COVID-19 pandemic and mental health consequences: Systematic review of the current evidence. Brain, behavior, and immunity, 89, 531-542. https://doi.org/10.1016/j. bbi.2020.05.048 